\title{
Parsing surrounding space into regions
}

\author{
NANCY FRANKLIN and LINDA A. HENKEL \\ State University of New York, Stony Brook, New York \\ and \\ THOMAS ZANGAS \\ New York University, New York, New York
}

\begin{abstract}
Surrounding space is not inherently organized, but we tend to treat it as though it consisted of regions (e.g., front, back, right, and left). The current studies show that these conceptual regions have characteristics that reflect our typical interactions with space. Three experiments examined the relative sizes and resolutions of front, back, left, and right around oneself. Front, argued to be the most important horizontal region, was found to be (a) largest, (b) recalled with the greatest precision, and (c) described with the greatest degree of detail. Our findings suggest that some of the characteristics of the category model proposed by Huttenlocher, Hedges, and Duncan (1991) regarding memory for pictured circular displays may be generalized to space around oneself. More broadly, our results support and extend the spatial framework analysis of representation of surrounding space (Franklin \& Tversky, 1990).
\end{abstract}

How do observers conceive of the space around themselves? One possible way is analytically, according to a set of Cartesian axes and regions. By such a scheme, regions would have equal status, would be of equal size (e.g., mutually exclusive $90^{\circ}$ quadrants or overlapping $180^{\circ}$ hemispheres), and would be represented with equal precision. For the relatively simple case in which a picture of a circle is presented within one's field of view (Huttenlocher, Hedges, \& Duncan, 1991), subjects have been argued to "parse" the figure into four mutually exclusive $90^{\circ}$ regions that seem not to differ in the precision of their representation. But this is unlikely to hold for surrounding space; previous work has shown that not all directions around oneself have equal status in memory (Franklin \& Tversky, 1990; Maki, Maki, \& Marsh, 1977; Shepard \& Hurwitz, 1984). Instead, their status derives from typical interactions of observers with space.

Specifically, subjects appear to impose a spatial framework of egocentric axes (front/back, left/right, head/feet) onto both described and physically surrounding space (Bryant, Tversky, \& Franklin, 1992; Franklin \& Tversky, 1990; Franklin, Tversky, \& Coon, 1992; Hintzman, O’Dell, \& Arndt, 1981). Within the spatial framework, the axes can be compared with respect to their perceptual, behavioral, and physical asymmetries, which are thought to reflect important functions that have evolved to favor particular directions (Clark, 1973). For the horizontal plane surrounding upright observers, the front is favored because movement, manipulation of objects, and percep-

We thank Barbara Tversky, G. A. Radvansky, Laura Carlson Radvansky, Ruth Maki, Doug Hintzman, and an anonymous reviewer for their helpful comments. Correspondence concerning this paper should be sent to Nancy Franklin, Department of Psychology, State University of New York, Stony Brook, NY 11794-2500 (e-mail: nfranklin@ccmail.sunysb.edu) tion are directed primarily frontward. (See also Sholl's, 1987 , discussion of the front as an "orienting schema.") Such important asymmetries on front/back give it precedence over right/left, as supported by faster front/back than right/left retrieval times (de Vega, 1994). Furthermore, the precedence of the frontward direction has been implicated by faster retrieval times to it than to other horizontal directions, including back. This has been found when subjects are identifying objects in a specified direction from themselves (e.g., Bryant et al., 1992; Franklin \& Tversky, 1990; Hintzman et al., 1981), when they are identifying positions on a compass rose, whose axes appear to be conceptualized with respect to egocentric axes (Loftus, 1978), and when they answer questions about the relative positions of objects in an array (Attneave \& Farrar, 1977).

Most of the research in this area has focused on the canonical directions constituting the spatial framework, with obliques sometimes included. The organization of the remaining $356^{\circ}$ of horizontal space is not as well understood. If the spatial framework is a general and powerful organizational structure for representing space, its influence should extend to the regions surrounding canonical directions. The current studies test the possibility that characteristics of entire regions can be predicted from considerations of the spatial framework. If the four canonical directions on the horizontal differ in their relative salience, then the regions they define should likewise differ.

We examine two characteristics by which regions are predicted to differ. The first is region size. Front, back, right, and left serve to divide horizontal space into four useful categories, but these categories need not be divided evenly. For the four poles, and the space immediately surrounding each, directions belong fairly unambiguously to a single category: front, right, back, or left. For other directions, the associated category or categories are less 
clear; directions that fall farther from $0^{\circ}, 90^{\circ}, 180^{\circ}$, and $270^{\circ}$ could plausibly be conceived of as being associated with more than one of them. We argue that it is useful to conceive of such directions as belonging to the category for which interactions with the world are functionally or perceptually more salient, even if they are physically closer to a less salient pole. This leads to two predictions. First, if the task does not force subjects to categorize space into mutually exclusive regions, we should observe some overlap in conceptual regions. Second, the more salient direction defining a quadrant (e.g., front) should capture more space within the quadrant than should the less salient direction (e.g., right). So, the functionally and perceptually most salient region, front, should be conceptually the largest. Right and left should not differ in size from one another.

Predictions for back are less certain. Access to information about the back is faster than it is about the left and right but slower than it is about the front (Bryant et al., 1992; Franklin \& Tversky, 1990; Hintzman et al., 1981; Sholl, 1987). But the source of this relatively high accessibility is not yet clear. If back's privileged status in previous studies is due solely to its association with and high discriminability from front, then the back region may conceptually be no larger than left or right. On the other hand, if its accessibility reflects the relative salience of the back itself, independent of its association to the front, properties such as size should fall intermediately between front and right/left.

The second characteristic we consider is discriminability in memory for various directions within a region. We will refer to this as a region's resolution. The greater the functional salience of a region, the more important it is for the observer to interact with, discriminate among, and remember the locations of various objects within it. Because movement, manipulation, and inspection are typically directed toward objects at or near the front, it should have the greatest resolution.

In the current studies, we measure resolution in two ways. The first is the accuracy with which subjects locate an object's former position. For similar tasks using pictures of circles and dot stimuli appearing on diameter lines, distance from landmarks such as region boundaries is a strong predictor of error, but which region is involved does not seem to matter (Huttenlocher et al., 1991; Nelson \& Chaiklin, 1980). The space used in the current studies, however, is quite different from those of Huttenlocher et al. and Nelson \& Chaiklin. Space surrounding oneself has psychologically important properties that pictures do not have (except, perhaps, maps and compass roses, which depict environments). The difference between the current situation and those of Huttenlocher et al. and Nelson and Chaiklin is accentuated by the task. The current studies require subjects to encode and remember spatial position with respect to themselves, whereas tasks involving memory for locations in pictures typically test for absolute location. Finally, surrounding space is not perceptually available in its entirety at any moment, while a picture of a circle is.
All of these considerations lead us to predict that the resolution of surrounding space will depend critically on one's orientation within it. Extending from previous findings and from the spatial framework analysis, we predict subjects' memory for direction to be greatest for objects located in the front region. Right and left should be worse and roughly equal to each other. If back is more salient than left and right, independent of its association with front, then recall performance should be intermediate between front and left/right. Otherwise, we should find no better performance in the back region than in the left or right.

Our second method for studying resolution is to examine subjects' use of directional terms in describing locations. It is reasonable to expect that representation of space in memory is reflected in the language used to describe it (e.g., Levelt, 1984). Two competing approaches are considered here. The first is that the main difference that will occur for the various regions is in the use of language to categorize space into gross regions. That is, a direction label will be used in proportion to the size of the region defined by that direction. Because front is predicted to be the largest region, it is predicted to be used most often in describing space.

The second approach makes just the opposite prediction. Degree of resolution in a memory representation should be reflected not by the frequency with which a term is used as a primary descriptor, but by the degree of detail given in the description. Descriptions of two positions within a more highly resolved region should be, on average, more discriminable than those for positions equally far apart in a less highly resolved region. Descriptions are made more discriminable, or more informative, by the use of linguistic hedges. So we would predict more hedges for positions within the front region than for positions within any other. Some of the most useful hedges available are the terms for adjacent regions. For instance, an object may be located a little to the right of front. So, if front is more highly resolved in memory than are left and right, "left" and "right" should frequently be used both for directions within those regions and for distinguishing among the various directions within the front. In contrast, "front" should be used as a hedge for left or right much less frequently and should be used primarily within its own boundaries. Because front is predicted to receive the greatest number of hedges, and because "left" and "right" are predicted to constitute a large proportion of them, "left" and "right" are predicted to be used most often, followed by "back," followed by "front."

\section{EXPERIMENT 1 Size of Regions Around Oneself}

Experiment 1 was designed to assess the region sizes associated with front, back, right, and left. This was accomplished indirectly, by asking subjects to point to the boundaries between adjacent regions. If the spatial framework analysis generalizes from egocentric direc- 
tions to regions, and if it extends beyond predicting retrieval times to predicting size, the greater functional salience of the front should render it conceptually largest.

\section{Method}

Subjects. Forty-one undergraduates (16 men and 25 women) at the State University of New York at Stony Brook participated for credit in a psychology course. All were right-handed.

Materials. Subjects sat in a homogeneous circular "room" $(1.68 \mathrm{~m}$ in diameter and $2.44 \mathrm{~m} \mathrm{high}$ ) with no environmentally defined front, back, or sides. The "room" was hung from a laboratory ceiling and consisted of a circular metal hoop onto which an opaque blue curtain was attached. The curtain extended between ceiling and floor, both of which were covered by solid green felt. The subject sat on a low, round, backless seat in the center of the "room" and $13 \mathrm{~cm}$ off the floor. The seat swiveled freely and rested on a single solid base centered beneath it. A $360^{\circ}$ prot ractor was printed on the floor surrounding the outside of the curtain, out of the subject's view.

Procedure. The subjects were given $30 \mathrm{sec}$ in which to swivel on the seat and acquaint themselves with the room. They then received 80 trials in which they pointed to region boundaries. The trials were organized into 10 blocks of 8 trials. At the beginning of each block, the subjects were oriented to face a randomly selected direction by aligning themselves with a rod $(1.3 \mathrm{~cm}$ in diameter $)$ that was slid under the curtain by the experimenter. Once the subject faced the designated direction, the experimenter removed the orienting rod and specified the hand in which the subject was to hold the response pointer ( $107 \mathrm{~cm}$ long and $.63 \mathrm{~cm}$ in diameter). If a trial involved a boundary on the right side, the subjects were told to hold the pointer in the right hand. If the trial involved the left side, they were to hold the pointer in the left hand.

The subjects responded to instructions of the form, "Point as far to the $X$ as possible so that you would still consider yourself as pointing to the $Y$ " ( $X$ and $Y$ were replaced by the direction terms "front," "right," "back," and "left," and referred to adjacent regions.) The subjects were told to hold the pointer at arm's length directly to the $Y$. They then were to indicate their responses by moving the pointer to the desired position and extending its tip out below the curtain, with the tip protruding from the "room." The experimenter could read the responses, to within $1^{\circ}$, off the floor protractor outside the "room." When making their responses, the subjects were allowed to turn their heads and shoulders but not the rest of their bodies.

After each block of eight trials, the subjects were reoriented to another, randomly determined, direction in order to keep any part of the room itself from acquiring special status during the course of the session. They then received another block of trials.

Design. Each subject made 10 placements for all eight $X-Y$ boundaries (front-right, right-front, etc.) Within each of 10 blocks, all eight boundaries were probed in random order.

\section{Results}

Data from 3 subjects ( 2 men and 1 woman) were eliminated because $25 \%$ of their mean responses were more than $10^{\circ}$ beyond the Cartesian hemisphere defined by the probed direction (e.g., a mean response of $190^{\circ}$ for right). For the remaining 38 subjects, eight mean boundary locations were calculated. A boundary was defined as the subject's mean response for a given $X-Y$ direction pair (where the subject's front is designated as $0^{\circ}$, right as $90^{\circ}$, back as $180^{\circ}$, and left as $270^{\circ}$ ). The results are shown in Table 1 and Figure 1.

The first column of Table 1 shows that the sizes of the regions described by these boundaries differ markedly
Table 1

Characteristics of Conceptual Region Boundaries (Experiment 1)

\begin{tabular}{lrcrrr}
\hline Direction & Size & $\begin{array}{c}S E \text { of } \\
\text { Region Size }\end{array}$ & Boundaries & \multicolumn{2}{c}{$\begin{array}{c}\text { Distance From } \\
\text { Defining Pole }\end{array}$} \\
\hline Front & $124^{\circ}$ & 5.5 & $299^{\circ}$, & $63^{\circ}$ & $61^{\circ}, 63^{\circ}$ \\
Back & $110^{\circ}$ & 6.4 & $125^{\circ}$, & $235^{\circ}$ & $55^{\circ}, 5^{\circ}$ \\
Right & $92^{\circ}$ & 5.3 & $43^{\circ}, 135^{\circ}$ & $47^{\circ}, 45^{\circ}$ \\
Left & $91^{\circ}$ & 6.4 & $226^{\circ}$ & $317^{\circ}$ & $44^{\circ}, 47^{\circ}$ \\
\hline
\end{tabular}

$[F(3,111)=9.08, p<.00005]$. Mean sizes were as follows: front, $124^{\circ}$; back, $110^{\circ}$; right, $92^{\circ}$; left, $91^{\circ}$ ). Front was significantly larger than both right and left (by Newman-Keuls post hoc $t$ tests) and nonsignificantly larger than back. Back was larger than right, and right and left did not differ reliably from each other. Standard errors for the various regions (second column) did not differ reliably.

Regions were symmetric about their poles (third and fourth columns). Boundaries for front were $61^{\circ}$ and $63^{\circ}$ from the canonical pole and correlated within subjects $[r(36)=.77, p<.001]$. Average deviation on either side of the canonical pole and the correlations between these deviations were as follows for the other regions: for back, $55^{\circ}$ and $55^{\circ}[r(36)=.88, p<.001]$; for right, $47^{\circ}$ and $45^{\circ}[r(36)=.43, p<.01]$; for left, $44^{\circ}$ and $47^{\circ}$ $[r(36)=.56, p<.001]$. In no case did absolute deviation from the canonical pole differ significantly for the two boundaries of a region. As can be seen from Table 2, the symmetry in the data was primarily defined with respect to canonical poles. That is, correlations for front-right and front-left (.77), for right-front and right-back (.56), for back-right and back-left (.88), and for left-back and left-front (.56) were all highly significant. Other possible patterns of symmetry [e.g., between the right boundary of front and the right boundary of back, with $r(36)=$ .23] did not consistently emerge.

\section{Discussion}

All regions, regardless of size, seemed to be defined with respect to their corresponding canonical pole $\left(0^{\circ}\right.$, $90^{\circ}, 180^{\circ}$, and $270^{\circ}$ ), as indicated by their symmetry about it. This lends validity to our characterization of surrounding regions as organized according to the four canonical directions.

For the relatively simple case in which a picture of a circle is presented within one's field of view, subjects have been argued to "parse" the figure into four mutually exclusive $90^{\circ}$ regions. For space around oneself, however, regions differ in size, some by more than $30^{\circ}$. Subjects appear to conceptualize right and left as quadrants with boundaries at or near the obliques $\left(45^{\circ}, 135^{\circ}, 225^{\circ}\right.$, and $315^{\circ}$ ), but front and back are larger. These findings are consistent with the spatial framework analysis (Franklin \& Tversky, 1990) and indicate that the nature of one's typical interactions with space predicts how subjects conceptualize it. As predicted, the most salient region emerged as largest.

Also, in contrast to how subjects are argued to represent circles, the sizes of the four regions around oneself 


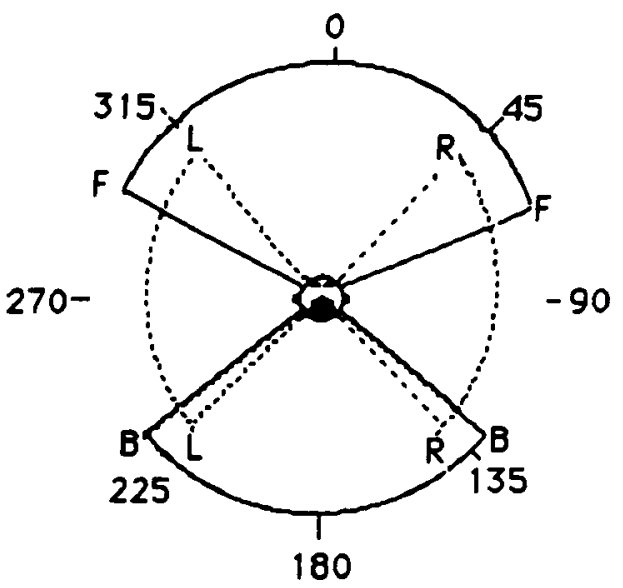

Figure 1. Mean boundary locations of eight conceptual regions around oneself (Experiment 1).

sum to more than $360^{\circ}$. That is, an $X-Y$ boundary is not the same as a $Y-X$ boundary. Subjects deviate further, for example, from the front-right oblique in order to define the boundary of front than they do in order to define the boundary of right. This may stem from uncertainty of boundary locations, but the magnitude of overlap suggests that it is likely not the only cause. It is not necessary that conceptual space be Cartesian space, with each location belonging uniquely to one region. More important is that any regional category with which a position is associated should be useful to the observer. Some parts of space, particularly near obliques, are included in more than one region. Surrounding space, then, might be represented categorically, but the categories are fuzzy.

\section{EXPERIMENT 2 Memory for Location Around Oneself}

We are capable of representing space not only categorically, but also more precisely. If we put an object down and want to retrieve it later, we should know not only that it is in a particular spatial region, but also where in the region it is. Particularly if we have turned or moved in the meantime, it would be useful to have encoded some absolute spatial cues indicating the position of the object. But it is also valuable to encode the relative po- sition of the object and oneself. This information is important for guiding motor responses, encoding the positions of items subsequently introduced, and updating the relative positions of the object and oneself. Experiment 2 examined memory for the precise relative position of an object as a function of the region in which it was originally placed.

Our primary prediction stemmed from our reasoning that a region of greater salience would be represented with greater accuracy. This leads to the prediction that front will have the lowest absolute error. Note that the largest region, and thus the one with the greatest opportunity for within-category error, is predicted to be recalled most precisely. This is sensible, given that the objects one is most likely to interact with or to explore perceptually are located toward the front, and thus one's ability to recollect their positions is most critical in this region.

\section{Method}

Subjects. Twenty-two right-handed undergraduates ( 15 men and 7 women) at SUNY Stony Brook participated in partial fulfillment of a course requirement.

Procedure. The apparatus was the same as that used in Experiment 1 . At the beginning of each trial, a round wooden peg $(3.2 \mathrm{~cm}$ in diameter, covering $2^{\circ}$, and $13.9 \mathrm{~cm}$ high) was placed by the experimenter in a target direction from the subject. The subject viewed it for $5 \mathrm{sec}$ and was allowed to turn his or her head and shoulders, but not the rest of the body, in order to see it. The peg was then removed, and the subject was instructed to reorient to a new, randomly determined, direction. Using the pointer used in Experiment 1 , the subject was to point in the direction from him- or herself in which the peg had originally been located. For example, if the object had been directly to the subject's right on initial presentation, the subject should point directly to his or her right, despite the intervening reorientation. Every $5^{\circ}$ around the subject were probed, excluding the four poles. Each position was probed twice, and positions were probed in random order. The subjects received three practice trials before beginning the test trials.

\section{Results}

Data greater than $3 S D$ from the mean response for each position ( $1.5 \%$ of the data) were eliminated.

In an effort to equate size of all regions under study, all comparisons were over $90^{\circ}$ areas symmetric about the canonical poles. Means for individual positions are shown in Figure 2.

Absolute error. If subjects conceive of space as homogeneous, the magnitude of error should be constant over

Table 2

Correlations Among Boundary Distances From Region's Defining Pole (Experiment 1)

\begin{tabular}{|c|c|c|c|c|c|c|c|c|}
\hline & \multicolumn{2}{|c|}{ FRONT } & \multicolumn{2}{|c|}{ RIGHT } & \multicolumn{2}{|c|}{ BACK } & \multicolumn{2}{|c|}{ LEFT } \\
\hline & $\begin{array}{c}\text { Front- } \\
\text { Left }\end{array}$ & $\begin{array}{l}\text { Front- } \\
\text { Right }\end{array}$ & $\begin{array}{c}\text { Right- } \\
\text { Front }\end{array}$ & $\begin{array}{l}\text { Right- } \\
\text { Back }\end{array}$ & $\begin{array}{l}\text { Back- } \\
\text { Right }\end{array}$ & $\begin{array}{c}\text { Back- } \\
\text { Left }\end{array}$ & $\begin{array}{l}\text { Left- } \\
\text { Back }\end{array}$ & $\begin{array}{l}\text { Left- } \\
\text { Front }\end{array}$ \\
\hline $\begin{array}{l}\text { F-L } \\
\text { F-R } \\
\text { R-F } \\
\text { R-B } \\
\text { B-R } \\
\text { B-L } \\
\text { L-B }\end{array}$ & & $.77 \ddagger$ & $\begin{array}{l}.43 \dagger \\
.59 \ddagger\end{array}$ & $\begin{array}{l}.51 \dagger \\
.56 t \\
.43 \dagger\end{array}$ & $\begin{array}{c}-.03 \\
.23 \\
.49 \dagger \\
.42 \dagger\end{array}$ & $\begin{array}{c}.10 \\
.37 * \\
-.06 \\
-.06 \\
.88 t\end{array}$ & $\begin{array}{l}.03 \\
.13 \\
.33^{*} \\
.31 \\
.77 \ddagger \\
.26\end{array}$ & $\begin{array}{r}-.13 \\
.25 \\
-.05 \\
-.15 \\
.31 \\
.89 \\
.56\end{array}$ \\
\hline
\end{tabular}



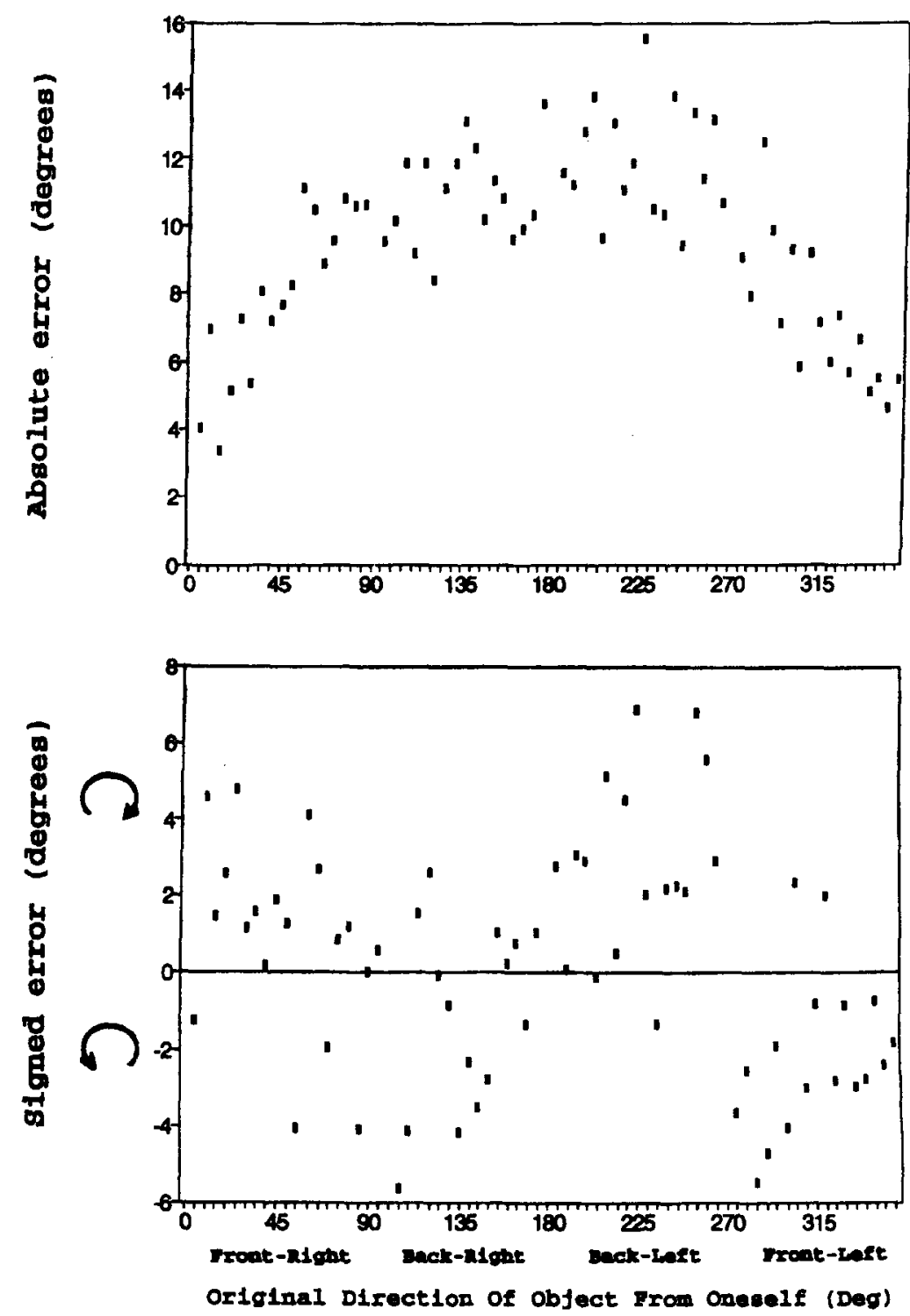

Figure 2. Accuracy of memory for direction around oneself (Experiment 2). The top panel shows the average magnitude of error (in degrees), regardless of its direction, as a function of position around oneself. The bottom panel shows average error (in degrees), taking direction into account.

different regions. Alternatively, if the conceptual properties of regions are predicted by the spatial framework analysis, the most salient region, front, should be most highly resolved and thus be most accurately recalled. We compared these predictions with a repeated measures analysis of variance (ANOVA) on the mean magnitude of errors, using region as the independent variable. The top panel of Figure 2 shows these means for all positions. The analysis was highly significant $[F(3,63)=35.76$, $p<.00005]$. By Newman-Keuls comparisons, front $\left(315^{\circ}-\right.$ $45^{\circ}$ ) was more accurate (mean error $=5.98^{\circ}, S E=.27$ ) than right $\left(45^{\circ}-135^{\circ}\right.$, mean error $\left.=10.23^{\circ}, S E=.45\right)$, left $\left(225^{\circ}-315^{\circ}\right.$, mean error $\left.=10.18^{\circ}, S E=.55\right)$, and back $\left(135^{\circ}-225^{\circ}\right.$, mean error $\left.=11.43^{\circ}, S E=.63\right)$. In addition, standard error for the front was smaller than for back $[F(21,21)=5.47, p<.001]$, right $[F(21,21)=2.72, p<$ $.05]$, and left $[F(21,21)=4.19, p<.005]$, with no other significant pairwise differences. That is, not only was mean absolute error smaller for front, but the range of responses was more limited for the front than for any other $90^{\circ}$ region.

Region landmarks (centers and boundaries) define the regions to which individual positions belong, and we have seen that the relative salience of these landmarks appears to predict mean error over the regions. But these landmarks might also predict error for individual positions within each region. Specifically, space may be more highly resolved and mean absolute error may be 
lower as proximity to these landmarks increases (Huttenlocher et al., 1991). Regression analyses on absolute error over all probed positions were conducted, with the following predictors: absolute deviation from the nearest pole in degrees, whether the initial location was within the front $\left(271^{\circ}-89^{\circ}\right)$ or back $\left(91^{\circ}-269^{\circ}\right)$ hemisphere, and whether initial location was on the right $\left(1^{\circ}-179^{\circ}\right)$ or left $\left(181^{\circ}-359^{\circ}\right)$ side. The only significant predictor of absolute error was the front/back variable $[t(66)=7.82$, $p<.001, S E=2.7]$, where absolute error is predicted by 11.4-3.77a $(a=1$ in the front hemisphere and 0 in the back hemisphere). That is, magnitude of error was smaller in the front $\left(0^{\circ}-89^{\circ}\right.$ and $\left.271^{\circ}-359^{\circ}\right)$ than in the back $\left(91^{\circ}-269^{\circ}\right)$, as we saw above, but overall, it was not predicted by distance from the nearest pole (front, right, back, or left).

Given the significance of front/back, and given the shape of the top panel of Figure 2, we conducted a separate analysis on absolute error using discrepancy in degrees from the front pole as a predictor. The analysis was significant $[t(66)=10.53, p<.001, S E=1.7]$, with absolute error predicted by $9.2+.01 b$ ( $b$ ranging from 5 to 175 and reflecting absolute discrepancy of original placement from the front pole). That is, absolute error increased as a function of distance from the front pole. As can be seen from the top panel, most of the effect occurred between $315^{\circ}$ and $45^{\circ}$, implying that the resolution of space is most sensitive to this factor within the front region.

Consistent with the spatial framework, we conducted the previous analyses as if region centers were at or near the $0^{\circ}, 90^{\circ}, 180^{\circ}$, and $270^{\circ}$ poles. If, instead, subjects construe space as a set of Cartesian quadrants (e.g., $0^{\circ}-90^{\circ}$, $90^{\circ}-180^{\circ}$, etc.), absolute error should be a function of deviation from the nearest oblique. For no oblique was this true. (This can be seen by examining the top panel of Figure 2 in $90^{\circ}$ segments centering around $45^{\circ}, 135^{\circ}, 225^{\circ}$, and $315^{\circ}$. The data are not symmetric around these obliques.)

Signed error. The overall trend that accuracy increases with proximity to the front is informative but incomplete; it does not tell us whether error is biased in particular directions as a function of location around oneself. We therefore examined the magnitude and valence (clockwise or counterclockwise) of error as a function of position around oneself. These means are presented in the bottom panel of Figure 2 .

As was true for absolute error, only the front/back factor successfully predicted the overall data for signed error. Average error at each position, where counterclockwise errors are depicted as negative and clockwise errors are depicted as positive, was analyzed by regression, and the least-squares fit to the data was described by the equation $y=-.10+.01 a-.74 b-.18 c$. In this equation, $a$ refers to discrepancy in degrees from the nearest pole, $b$ to the value on front/back $(b=1$ if the target was originally placed in the front hemisphere and -1 if placed in the back hemisphere), and $c$ to the value on right/left ( $c=1$ for right and -1 for left). The front/back factor was significant $[t(66)=2.06, p<.05, S E=3.6]$.
Inspection of the bottom panel of Figure 2 suggests that error as a function of position on the $360^{\circ}$ scale would follow a cubic pattern, in contrast to the inverted U-shape pattern for absolute error. The fit to a cubic function is significant $[F(1,66)=4.75, p<.05]$. The data are predicted by the formula $y=3.46-.93 x+.0007 x^{2}-$ $1.29 x^{3}$, and the pattern of signed errors can be described with respect to regions. Errors tended to be clockwise from $0^{\circ}$ to $90^{\circ}$, counterclockwise from $90^{\circ}$ to $180^{\circ}$, clockwise from $180^{\circ}$ to $270^{\circ}$, and counterclockwise from $270^{\circ}$ to $360^{\circ}$. Direction of error shifted from counterclockwise to clockwise, or vice versa, near $0^{\circ}, 90^{\circ}, 180^{\circ}$, and $270^{\circ}$.

To better understand this pattern of data, we conducted regressions separately for each of the $90^{\circ}$ regions centered at the four poles. We tested for all of the predictors reported previously (side of pole on which an object was originally placed, absolute angular distance of original placement from the front, and absolute angular distance of original placement from the nearest pole). For front, back, and left, only the side of the pole on which the object was originally placed significantly predicted the data. For front, $y=.22+1.68 c[t(16)=4.03, p<.001$, $S E=1.8$, where $c$ codes whether original placement had been on the right or the left side of the pole. That is, errors were clockwise on the right side of front $\left(1^{\circ}-44^{\circ}\right)$ and counterclockwise on the left side of front $\left(316^{\circ}-\right.$ $\left.359^{\circ}\right)$. For back, $y=.82-2.05 c[t(16)=3.88, p<.001$, $S E=2.2]$. Again, errors for the right side of the back region $\left(136^{\circ}-179^{\circ}\right)$ were generally toward the right, and those on the left side $\left(181^{\circ}-224^{\circ}\right)$ were generally toward the left. For the left region $\left(226^{\circ}-314^{\circ}\right), y=.31-2.96 b$ $[t(16)=5.00, p<.001, S E=2.5]$, where $b$ denotes whether original placement had been on the front or back side of the pole. Within this region, errors were biased away from the front and back poles and toward the left. Regression analyses for the right region were nonsignificant.

Oblique-centered analyses similar to those described above were performed for all regions, and none were significant.

\section{Discussion}

Subjects reoriented between encoding and retrieval, and thus the findings pertain to relative, not absolute, spatial relations. We found subjects to be quite capable of performing a task that required them to encode spatial relations with respect to themselves and independent of absolute positioning in the room. When they do this, however, their responses follow a predictable pattern of representational bias: The accuracy with which they encode a position depends on its angular disparity from $0^{\circ}$. Overall, absolute error was predicted by discrepancy of initial position from an important landmark, following Huttenlocher et al. (1991; see also Hayward \& Tarr, in press), but the only consistent landmark was the front pole (see also Venturino, 1992).

When we consider the valence of the errors as well, we see a more complex pattern. In none of our analyses was signed error a simple function of discrepancy of target 
position from the nearest pole. A categorical effect did, however, emerge. Errors near the front and back poles were biased away from these referent poles and on the same side as the original placement, reminiscent of Huttenlocher et al. (1991). For example, if original placement was slightly to the right of the front pole, responses tended to be biased further to the right of the original position; if original placement was slightly to the right of the back pole, responses were similarly biased toward the right (i.e., toward $90^{\circ}$ ). This pattern suggests, according to the category model of Huttenlocher et al., that the front and back poles may serve as boundaries at which sampling errors in memory are truncated. That is, the front-back axis may provide important, well-defined referent directions with respect to which positions are encoded in memory. One stable piece of information in memory, the categorical relationship of the target position and the referent axes, would affect the acceptable range of error with which uncertain precise positions can be sampled. In fact, such a view is consistent with other findings in the literature, such as tilt contrast in the reproduction of real or virtual lines in frames (Beh, Wenderoth, \& Purcell, 1971). A target line that is near the $x$ or $y$ axis, for example, is later misremembered as angularly farther away from it (Bryant \& Subbiah, 1993).

Alternatively, the finding can be explained by an attraction effect of the right and left poles. This possibility is consistent with the literature on anchor effects, which are produced when a spatial target is encoded inaccurately and memory is biased toward a salient location or frame of reference (Nelson \& Chaiklin, 1980; Sadalla, Burroughs, \& Staplin, 1980; Schiano \& Tversky, 1992; Tversky, 1981; Wenderoth, Parkinson, \& White, 1979). Both possibilities would ensure that offcenter objects are coded in memory on the appropriate side of the front or back pole and would provide a mechanism for maintaining high discriminability among locations near these poles. Because the effect was observed for positions near both the front and back poles, the current data do not strongly favor either interpretation over the other.

An answer to the question of the back's status remains elusive. In previous work, subjects have been found to be more accurate in recalling that objects had been in the back region than in recalling that objects had been in the left or right region (Hintzman et al., 1981). However, those subjects were not asked to recall precise positions, only categorical directions. The current experiment compared memory for precise position within each region, and the results do not suggest a relative advantage for back. The results for signed error, however, suggest that $180^{\circ}$ may serve as a boundary beyond which sampling errors are truncated; locations were rarely misremembered as falling on the wrong side of it. On the other hand, we can say with confidence that back has a less privileged status than front. Like previous studies involving errors in pointing (Sholl, 1987) and times to judge categorical re- lations among items (Attneave \& Farrar, 1977), we find evidence that representation of the world behind oneself is cruder than, or secondary to, the world in front.

\section{EXPERIMENT 3 Describing Directions}

Using a retrieval task that involved pointing, Experiment 2 supported the primary status of the front. But if the front is more highly resolved than the other regions, this should show up with other tasks as well. It would be particularly impressive if the task did not require such fine motor movements as pointing, which may be easier in the front than elsewhere.

In Experiment 3, subjects viewed objects placed in various positions around themselves and described their directions so that another subject could duplicate the position of the objects. Two hypotheses were considered. The first was that use of spatial terms would vary directly with region size. By this reasoning, "front" would be used most often in descriptions, and its greater frequency should arise from areas near the obliques, where front extends into right and left, but not vice versa. However, this hypothesis predicts only the use of terms to convey the single canonical direction that subjects consider most closely to describe its position. Such gross information almost certainly will be used in describing the precise direction of an object, regardless of its location. So, upon closer inspection, this intuitively appealing argument makes its predictions without regard to possible differences in resolution among regions.

The alternative hypothesis is based on the use of directional terms as qualifiers of primary categorical information. It is in this secondary, qualifying capacity that differences are more likely to emerge, because the use of qualifiers is optional in a description and there is greater opportunity for variance in their use. This leads to the prediction that functionally more important regions will have higher resolution and that subjects' use of resolving details and hedges will reflect their importance. Moreover, terms referring to less salient and nearby directions will constitute many of these hedges. Because front is the most salient region, right and left should be invoked more often to qualify positions within it than vice versa.

\section{Method}

Subjects. Thirty-eight right-handed undergraduates (16 men and 22 women) at SUNY Stony Brook participated in this study for course credit.

Procedure. The experiment took place in the "room" used in Experiments 1 and 2. For each trial, subjects described the location of an object placed by the experimenter. A trial proceeded as follows: The experimenter slid a cylindrical peg (the one used in Experiment 2) immediately inside the curtain and stood it on end, and the subject had $5 \mathrm{sec}$ in which to study its location. The subject was allowed to turn his head and shoulders but not the rest of the body. Then the peg was removed, and the subject was told that there was another subject with a peg in an identical room nearby. (In fact, the other subject did not exist.) The subject's goal was to describe the 
egocentric position of the peg so that the "other subject" could place his peg in a direction around himself that matched as accurately as possible. The subjects' descriptions were recorded by a microphone that hung above their heads and was attached to a hidden tape recorder. The subjects were told that the microphone was attached to a speaker in the partner's room and that the partner could listen to the descriptions but could not provide feedback. Clockface and compass terms were not allowed, but adjective modifiers (e.g., "a hair to the left," "dead front," "halfway between back and left") were.

Design. Positions were probed at every $4^{\circ}$ around the subject $\left(0^{\circ}\right.$, $4^{\circ}, 8^{\circ}$, etc.), in random order. Each position was probed once. Trials were given in 10 blocks, with 9 trials per block. The subject was reoriented to a randomly determined position for each block.

\section{Results}

We used fairly liberal criteria for identifying direction terms. "Front," "ahead," "forward," and references to body parts that point forward (e.g., stomach, chest, face) were counted as referring to the front. Similarly, "back," "behind," "rear," "left," "leftward," "right," "rightward," and other terms that referred clearly to a particular side of the body were accepted as specifying directions.

We present our data for this experiment in several ways. In Table 3 and Figure 3, we show the point farthest from the defining pole at which a direction term was given at least $80 \%$ of the time. ${ }^{1}$ Use of right and left terms was not symmetric about the $90^{\circ}$ and $270^{\circ}$ poles. Instead, use of each was elongated toward the front. A repeated-measures ANOVA on the range over which subjects used a given term was highly significant $[F(3,102)=5.88, p<$ $.001]$. A more conservative analysis, which excluded the angularly most distant $20 \%$ of positions at which each term was used by a subject, was marginally significant $[F(3,102)=2.35, p<.08$.

First, we tested the hypothesis that frequency of use for the various terms is a direct function of the size of the primary category to which a position belongs. To do this, we analyzed descriptions that used only one term referring to spatial direction (i.e., "front," "back," "right," "left," or any other term that clearly referred to one of these categories). If the hypothesis holds, then the predicted effect should be most apparent in this subset of descriptions. An ANOVA comparing the frequency of use for these terms was significant $[F(3,111)=19.8$, $p<.0001]$. Although front emerged in Experiment 1 as the largest region, "front" (mean $=2.8$ instances) was used less often in single-direction descriptions than was "back" (7.0 instances), "right" (7.4 instances), or "left" ( 8.1 instances) $(p<.05$ in all cases by Newman-Keuls post hoc $t$ tests). Thus, the prediction of the first hypothesis was not supported, and in fact, the opposite finding

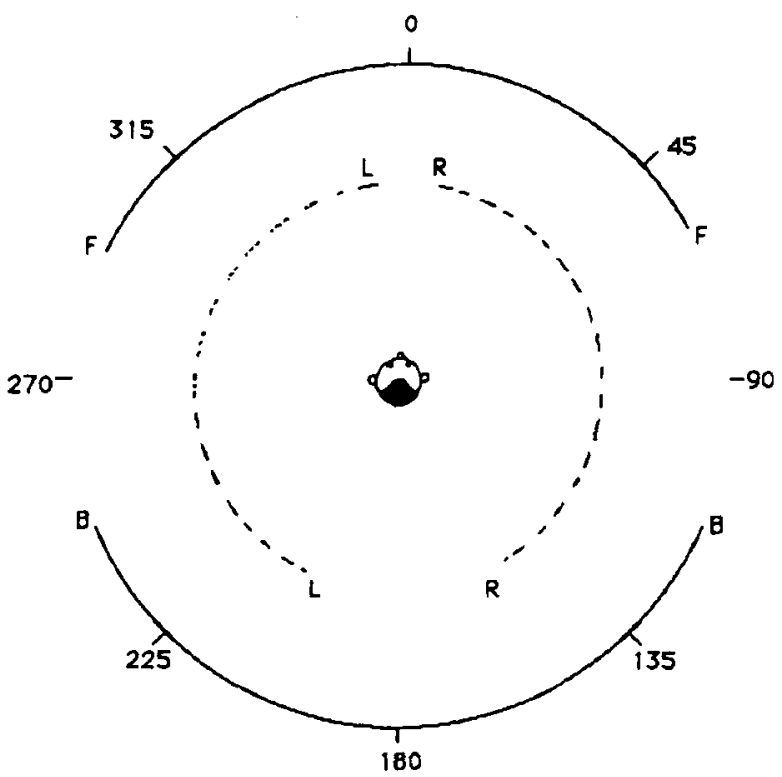

Figure 3. Furthest position at which $80 \%$ of responses included a given direction term (Experiment 3).

was obtained. Frequency of use for terms referring to back, left, and right did not differ reliably from each other. We will return to this interesting pattern later.

For descriptions that made use of two direction terms (e.g., "to the right of front"), two judges, blind to the purpose of the experiment, calculated the frequency with which terms occupied primary versus secondary positions in the description, independent of order of mention. For example, "front" is modified by "right" in the above example, and so "front" has the primary status. For this set of two-direction descriptions, mean frequencies with which terms had primary status were: $\mathbf{1 4 . 0}$ (front), 13.2 (back), 14.3 (right), and 14.3 (left). Mean frequencies with which they had secondary status were: 13.4 (front), 15.1 (back), 12.9 (right), and 14.3 (left). In an ANOVA, direction term was significant $[F(3,111)=7.14, p<.001]$, as was the interaction of primary/secondary status with direction term $[F(3,111)=8.84, p<.0001]$. Note, in particular, that when "front" was used in a description, it was more likely to occupy the primary or referent position than the secondary or qualifying position. This was not true for either "back" or "left," although it did unexpectedly occur for "right" as well.

In a fourth analysis, we sought to determine whether subjects use nonspatial qualifiers differently as a function of spatial position. We reasoned that this effect

Table 3

Furthest Position at Which $80 \%$ of Responses Include a Given Direction Term (Experiment 3)

\begin{tabular}{lccccrc}
\hline Region & $\begin{array}{c}\text { Extent of } \\
\text { Use }\end{array}$ & $\begin{array}{c}S E \\
\text { of Extent }\end{array}$ & $\begin{array}{c}\text { Difference from } \\
\text { Experiment 1 }\end{array}$ & Endpoints & $\begin{array}{c}\text { Dev from } \\
\text { Pole }\end{array}$ \\
\hline Front & $124^{\circ}$ & 6.9 & $5^{\circ}$ & $296^{\circ}$, & $60^{\circ}$ & $64^{\circ} \& 60^{\circ}$ \\
Back & $136^{\circ}$ & 6.1 & $24^{\circ}$ & $112^{\circ}, 248^{\circ}$ & $68^{\circ} \& 68^{\circ}$ \\
Right & $140^{\circ}$ & 12.9 & $48^{\circ}$ & $8^{\circ}, 148^{\circ}$ & $82^{\circ} \& 58^{\circ}$ \\
Left & $144^{\circ}$ & 10.6 & $61^{\circ}$ & $208^{\circ}, 352^{\circ}$ & $62^{\circ} \& 82^{\circ}$ \\
\hline
\end{tabular}


would emerge in a fine-grained analysis of position within regions rather than in a categorical analysis comparing the regions themselves. To keep the number of categories reasonably small, we divided the descriptions into four ranges that we felt reflected four broad classes of "extremity" values, from descriptions that imply slight tendencies in a particular direction to those implying very strong tendencies. We applied a 4-point scale to subjects' responses, assigning a value of 1 to descriptions with qualifiers such as "slightly" or "kind of," 2 to qualifiers such as "more to the $X$ than the $Y$," 3 to strong but not precise qualifiers such as "almost" or "a lot," and 4 to precise qualifiers such as "directly" and "completely." If subjects are more confident or more emphatic in their descriptions of locations in the center of a conceptual region, then the mean descriptor for that area should have a higher value on this scale than other parts of the region. A regression on this value was highly significant, with magnitude of deviation from the closest pole predicting degree of emphasis $[t(37)=12.8, p<.00001, S E=.07]$. Emphasis is predicted by $3.23-.02 a$ (where $a$ is magnitude of deviation in degrees from the nearest pole). That is, emphasis is highest at the poles and decreases as a function of the described location's distance from the nearest pole (Figure 4). Thus, use of nonspatial hedge terms also varied as a function of spatial position, with their use predicted by the canonical axes of the spatial framework.

\section{Discussion}

Two opposing hypotheses were considered. One predicted that "front" should be used to describe an object's position most of ten because of this region's greater size,

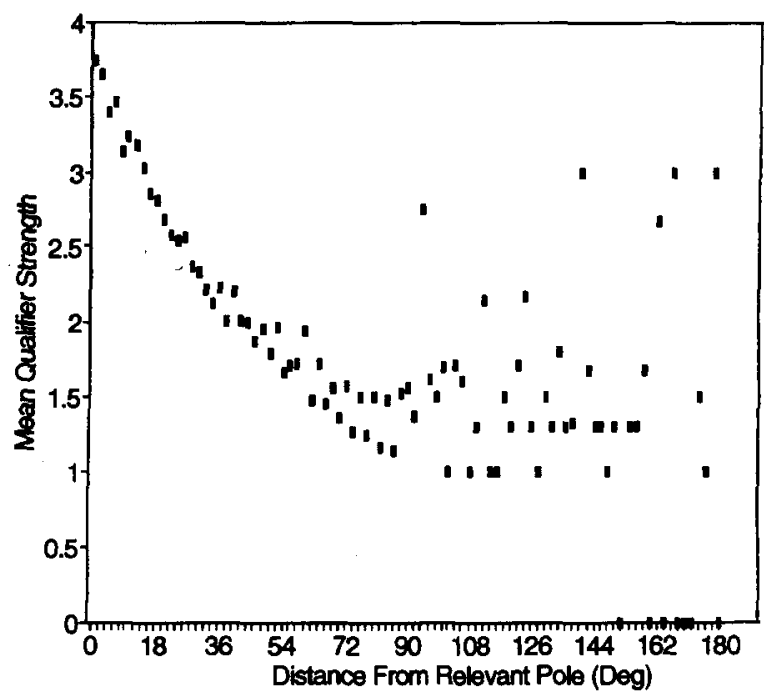

Figure 4. Mean strength of qualifier in the use of directional terms. A higher position on this scale indicates more emphatic use of a term. For example, a value of 1 would be assigned to "slightly to the right," 2 to "more to the right than the front," 3 to "almost directly to the right," and 4 to "directly to the right." A value of 0 indicates that no subject used the term at that position. as established in Experiment 1. The second was that the use of hedges in descriptions is a more accurate reflection of a region's resolution than is gross categorization of space. So "right" and "left" should be used most often, and primarily as hedges. In addition, use of "right" and "left" hedges should extend further into the more highly resolved front than into back.

Overall, the data were not consistent with the prediction that subjects' use of spatial terms would vary with the size of their corresponding regions. Rather, subjects described the different regions with different degrees of detail, and differences in the use of spatial terms seemed to lie primarily in their function as qualifiers for positions in adjacent regions. The use of "front" was confined almost completely to within the boundaries of the front region (where boundaries are defined by Experiment 1 results). The use of "back" extended $15^{\circ}$ in each direction beyond the boundaries of the back. "Right" and "left" were used over a broad and asymmetric range of positions, extending well outside their own boundaries, over nearly all of the front and much of the back. These findings reflect either different degrees of resolution in memory representation for the different regions or a bias in the degree of detail that subjects feel is optimal for communicating about the various parts of egocentric space (Grice, 1975). Given the results of Experiment 2, and given that the subjects' task was to provide as detailed descriptions as they could, we favor a representation-based explanation. Such an interpretation corroborates the strong case made by Landau and Jackendoff (1993) and others that the structure of language reflects the structure of spatial representation.

One very interesting finding was that the hypothesis predicting differences in gross categorical use couldn't even predict the data for descriptions involving only one directional term. A closer look at the data gives a clue as to why: In many of these descriptions, "front" was treated as a default and was not explicitly stated. For example, an object at $5^{\circ}$ may be (and was) described as simply "a little to the right." When a primary category term was absent and only a secondary term was present, the peg had always been placed in the subject's front region. This allows us to make sense of the relatively less frequent use of "front." Also, the tendency to give descriptions consisting of only single-direction modifiers for objects in the front region probably led to a reduction in the frequency with which "front" was used as the primary term in twodirection descriptions. This makes the significant ANOVA reported above more noteworthy.

Our more fine-grained regression analysis showed the average emphasis on direction labels to be a function of the target's angular departure from the relevant canonical pole. This effect is similar to the findings of Hayward and Tarr (in press), who used different materials and different methods. They showed subjects pictures in which a target object (e.g., a bird or fish) was positioned with respect to another object (e.g., a floating raft) and asked the subjects to either describe the spatial relationship or to judge descriptive sentences. In both tasks, preference 
for direction labels was a direct function of the angular proximity of the target to the relevant canonical pole emanating from the referent object. Thus, this seems to be a general effect of describing spatial directions between objects; it is not required that one of the objects be oneself or that the presented configuration have a particular size or dimensionality.

\section{GENERAL DISCUSSION}

The homogeneous environments used in the current experiments minimized the number of external cues (e.g., corners, walls, meaningful objects, and physical boundaries) usually available for conceptualizing space. With this information absent, subjects had to rely on egocentric cues, and their conceptualization of space was predicted by the way in which they typically interact with it. Subjects seemed to organize space into regions defined according to the front, back, left, and right poles, but these regions were not of equal size and status. Instead, the results for surrounding regions supports and extends what is known about differences between egocentric axes (Franklin \& Tversky, 1990).

The special status of front has long been argued for (e.g., Clark, 1973) and is well established both for previously viewed environments (e.g., Attneave \& Farrar, 1977; Hintzman et al., 1981; Sholl, 1987) and for environments learned through description (Bryant et al., 1992; Franklin \& Tversky, 1990; Franklin et al., 1992). Most studies have relied on retrieval or judgment time, with some studies in related literatures relying on accuracy measures (Huttenlocher et al., 1991; Loftus, 1978; Nelson \& Chaiklin, 1980). Using a different set of paradigms, the current studies support and extend the primary status of the front. In Experiment 1, subjects pointed to where they considered the outermost boundaries of the front, back, left, and right regions around themselves to be, and front emerged as the largest region. Front was found to be the most highly resolved of the four regions around oneself. Subjects recalled target directions with greatest precision for the front (Experiment 2), and directions in this region were described with the greatest discriminative detail (Experiment 3).

The current results do not, however, provide clear answers about the status of back. In Experiment 1, the size of back fell intermediately between the sizes for front and sides, and in Experiment 3, use of qualifiers for describing locations in the back fell intermediately between those for front and sides. These results are consistent with the interpretation that the salience of back is independent of its association and high discriminability from front. On the other hand, differences involving the back tended to be relatively small when found, and the question about its status in memory remains unresolved.

Nevertheless, we can conclude that a Cartesian characterization of four regions that are equal in size and status, while appropriate for pictures of relatively meaningless circular arrays (e.g., Huttenlocher et al., 1991;
Nelson \& Chaiklin, 1980), does not extend to the current situation. Subjects' responses for surrounding space indicated that their representation of it reflects differences in the importance of various directions to an acting, perceiving observer. It is sensible, then, that representation of pictures depicting space in which one can be embedded as an observer, such as visual maps and compass roses (Hintzman et al., 1981; Loftus, 1978), resembles the current situation more than it resembles memory for uninterpreted circles.

Although our findings for surrounding space depart from the typical findings for memory for pictures, we can still draw from models of picture memory to make sense of our data. According to Huttenlocher et al.'s (1991) category model, item location in circular displays seems to be coded at two levels of detail, categorical and finegrained. We find evidence for a similar means of representing surrounding space, with fine-grained effects tending to depend on position with respect to the front pole. In addition, region boundaries (corresponding to $0^{\circ}, 90^{\circ}, 180^{\circ}$, and $270^{\circ}$ ) have been argued to be the primary landmarks in circles (Huttenlocher et al., 1991). For space around oneself, region centers (i.e., the poles) are the primary landmarks, but these centers also occur at $0^{\circ}, 90^{\circ}, 180^{\circ}$, and $270^{\circ}$.

We have argued, on the basis of the spatial framework analysis, that the primary status of front stems in part from general perceptual differences and from biases in representation stemming from these differences. Is the primary status of front in these studies the result of perceptibility of the immediate situation? In visual perception, there is less locational information available for stimuli that are viewed in the periphery than for foveated stimuli, and objects currently toward one's front are typically foveated. This simple difference, however, is unlikely to account for the results here and in previous studies. First, front is privileged relative to other directions even when objects are not perceived (Franklin \& Tversky, 1990). Second, it is not clear why resolution of the visual field should predict front to be conceptually larger than the other regions (Experiment 1). Third, right, left, and much of back actually were easily foveated, since subjects turned their heads to examine the various regions of space. Front, back, left, and right of the subjects were defined with respect to the torso, not the eyes.

We do not mean to suggest with our current findings that we have identified the absolute boundaries of front, back, left, and right, or that we have determined their absolute resolutions. We interpret our results as, at best, indicating what these values are for the specific conditions under study (e.g., using the current distances, objects, tasks, etc.), although these values were chosen to be fairly typical (i.e., fist-sized objects at arm's distance). We are more interested in determining relative differences, and we believe that the current studies represent typical situations in which subjects interact with surrounding space under conditions of reduced external cues. We think that these studies provide an ap- 
propriate first look at the relative differences under investigation.

\section{REFERENCES}

Attneave, F., \& Farrar, P. (1977). The visual world behind the head. American Journal of Psychology, 90, 549-563.

Beh, H., Wenderoth, P., \& Purcell, A. (1971). The angular function of a rod-and-frame illusion. Perception \& Psychophysics, 9, 353-355.

BRyant, D. J., \& Subbiah, I. (1993). Strategic and perceptual factors producing tilt contrast in dot localization. Memory \& Cognition, 21 773-784.

Bryant, D. J., TVersky, B., \& Franklin, N. (1992). Internal and external spatial frameworks for representing described scenes. Journal of Memory \& Language, 31, 74-98.

Clark, H. H. (1973). Space, time, semantics, and the child. In T. E. Moore (Ed.), Cognitive development and the acquisition of language (pp. 27-63). New York: Academic Press.

DE VEGA, M. (1994). Characters and their perspectives in narratives describing spatial environments. Psychological Research, 56, 116-126.

FrankLin, N., \& TVERsky, B. (1990). Searching imagined environments. Journal of Experimental Psychology: General, 119, 63-76.

Franklin, N., TVersky, B., \& COON, V. (1992). Switching points of view in spatial mental models. Memory \& Cognition, 20, 507-518.

GRICE, H. P. (1975). Logic and conversation. In P. Cole \& J. L. Morgan (Eds.), Syntax and semantics: Vol 3. Speech acts (pp. 41-58). New York: Seminar Press.

HAYWARD, W. G., \& TARR, M. J. (1995). Spatial language and spatial representation. Cognition, 55, 39-84.

Hintzman, D., O'Dell, C., \& ARndT, D. (1981). Orientation in cognitive maps. Cognitive Psychology, 13, 149-206.

Huttenlocher, J., Hedges, L. V., \& Duncan, S. (1991). Categories and particulars: Prototype effects in estimating spatial location. Psychological Review, 98, 352-376.

LANDAU, B., \& JACKENDOFF, R. (1993). "What" and "where" in spatial language and spatial cognition. Behavioral \& Brain Sciences, 16, $217-265$.

LEVELT, W. (1984). Some perceptual limitations on talking about space.
In A. J. van Doorn, W. A. van de Grind, \& J. J. Koenderink (Eds.), Limits in perception (pp. 323-358). Utrecht: VNU Science Press. LofTUs, G. (1978). Comprehending compass directions. Memory \& Cognition, 6, 416-422.

MAKI, R., MAKI, W., \& MARSH, L. (1977). Processing locational and orientational information. Memory \& Cognition, 5, 602-612.

NeLSON, T., \& CHAIKLIN, S. (1980). Immediate memory for spatial location. Journal of Experimental Psychology: Human Learning \& Memory, 6, 529-545.

Sadalla, E. K., Burroughs, W. J., \& Staplin, L. J. (1980). Reference points in spatial cognition. Journal of Experimental Psychology: Human Learning \& Memory, 6, 516-528.

SCHIANO, D. J., \& TVERSKY, B. (1992). Structure and strategy in encoding simplified graphs. Memory \& Cognition, 20, 12-20.

SHEPARD, R. N., \& HURwiTZ, S. (1984). Upward direction, mental rotation, and discrimination of left and right turns in maps. In S. Pinker (Ed.), Visual cognition (pp. 161-193). Amsterdam: Elsevier.

SHOLL, M. J. (1987). Cognitive maps as orienting schemata. Journal of Experimental Psychology: Learning, Memory, \& Cognition, 13, 615-628.

TVERSKY, B. (1981). Distortions in memory for maps. Cognitive Psychology, 13, 407-433.

Venturino, M. (1992, April). A spatial position effect in ordered recall from spatial memory. Paper presented at the meeting of the Eastern Psychological Association, Boston.

Wenderoth, P., Parkinson, A., \& White, D. (1979). Comparison of visual tilt illusions measured by the techniques of vertical setting, parallel matching, and dot alignment. Perception, 8, 47-57.

\section{NOTE}

1. Using a $90 \%$ criterion produced very similar results to the $80 \%$ criterion. Subjects' responses were fairly consistent with one another. Thus, the point at which we chose our cutoff doesn't seem to make much of a difference.

(Manuscript received July 26, 1993; revision accepted for publication July 14, 1994.) 